\title{
ON THE ECLIPSING BINARIES IN THE GLOBULAR CLUSTERS NGC 3201 AND NGC 5139
}

\author{
E. H. GEYER \\ Astron. Institut der Universität Bonn, Observatorium Hoher List, F.R.G.
}

\begin{abstract}
V 65 is not a member of NGC 3201; there is no certainty about the membership of V 78 to NGC 5139 ( $\omega$ Cen).
\end{abstract}

The frequency and existence of double stars in old population systems like globular clusters is still unsolved. The observational approach for tackling this problem is to search for variable stars which are somehow related to duplicity, e.g. eclipsing binaries, U Geminorum stars and Novae. In the latest edition of the catalogue of variable stars in globular clusters by Sawyer Hogg (1973) only 10 variable stars are known to be eclipsing binaries, yet only three of them, all EA-types, are considered as possible cluster members (Sawyer Hogg, 1972; Kukarkin, 1973). These eclipsing binaries are V65 in NGC 3201 with a period of 1.66, V78 in NGC 5139 ( $\omega$ Cen) with $P=1.168$ and V3 in NGC 6838 (M71) with $P=3$.79. The latter cluster is according to Arp and Hartwick (1971) more like an old population I cluster, and only 3 other variables in the cluster region are known. The magnitude of V3/NGC 6838 suggest that its position in the C-M diagram is in the 'blue-straggler' sequence of this cluster.

On six nights in March this year I observed photoelectrically in $U B V$ the two other $E A$-binaries with the $1 \mathrm{~m}$ telescope of the European Southern Observatory. Some $60 U B V$ observations were obtained for V 65 in NGC 3201 and about $100 U B V$ measurements for V 78 in NGC 5139. Just a few weeks ago I also received an image tube spectrum in the red spectral region of V 65/NGC 3201 taken with the ESO $1.5 \mathrm{~m}$ reflector.

\section{Discussion}

\subsection{65 IN NGC 3201}

This globular cluster has a low galactic latitude $\left(+9^{\circ}\right)$, and its radial velocity is high and amounts to $+493 \mathrm{~km} \mathrm{~s}^{-1}$. A colour-magnitude diagram was given by Menzies (1967; Alcaino, 1973). The eclipsing variable V 65 is at quite a large angular distance, 16 '1, from the cluster center; yet close to its position are the RR Lyrae stars V 55, $\mathrm{V} 64$ and the red variable V 68 .

From a preliminary analysis of the photometric measurements of $\mathrm{V} 65$ at maximum light and 3 sporadic observations of the neighbouring star $\mathrm{V} 64$, the average values of Table I were derived.

The reddening of $\mathrm{V} 65$ amounts to about $E_{B-V} \sim 0^{m} 18$, and the photometrically determined spectral types are given in the last column of Table I. Obviously if V 65 is a cluster member, then its position in the Hertzsprung-Russell diagram is about 1 mag. 
TABLE I

Photometric data for V 64 and V 65 in NGC 3201

\begin{tabular}{llllllll}
\hline Variable & $\begin{array}{l}\text { Distance } \\
\text { from } \\
\text { Center }\end{array}$ & Period & Type & $V$ & $B-V$ & $U-B$ & $\begin{array}{l}\text { Sp. } \\
\text { Type }\end{array}$ \\
\hline V 64 & 16.0 & $0 ! 522$ & RRab & 14.52 & +0.50 & +0.23 & A 5: \\
V 65 & $16 ! 1$ & 1.660 & EA & 13.54 & +0.49 & +0.26 & A 5: \\
\hline
\end{tabular}

above the RR Lyrae gap of NGC 3201, which is not plausible. Actually the image tube spectrum $\left(180 \AA \mathrm{mm}^{-1}\right)$ on which 5 stellar lines can be measured not only confirms the spectral type but yields a radial velocity relative to the Sun of $v_{\mathrm{s}}=$ $=+83 \pm 19 \mathrm{~km} \mathrm{~s}^{-1}$, which is completely different from the cluster velocity of $+493 \mathrm{~km} \mathrm{~s}^{-1}$. Since V 65 was close to the secondary minimum phase when the spectrum was obtained, the orbital velocity cannot be blamed for this difference. V 65 is therefore a distant eclipsing binary not related to NGC 3201 .

\subsection{78 IN NGC 5139}

Different arguments for cluster membership of this 1.168-period eclipsing binary have been given: Dickens and Saunders (1965) state that its proper motion is indistinguishable from cluster members, and Geyer (1971a, 1973) gives its UV-excess which is comparable to that of the RR Lyrae variables of $\omega$ Cen.

The position of this eclipsing binary in the C-M diagram falls within the brighter domain of the RRc stars of the cluster (Geyer, 1966, 1970; Sistoro, 1968). A photoelectric investigation of this binary system has been presented by the author at the IAU Colloquium No. 15 (1971b). The newly obtained photometric observations seem to confirm the intrinsic variability of the smaller and hotter component which is occulted in the primary minimum.

\section{Acknowledgements}

I wish to thank Dr P. Åberg, Uppsala Observatory, for some photometric observations, and Dr J. Danziger, European Southern Observatory, for taking the spectrum of $\mathrm{V} 65$.

\section{References}

Alcaino, G.: 1973, Atlas of Galactic Globular Clusters with Colour Magnitude Diagrams, Universidad Católica de Chile.

Arp, H. C. and Hartwick, F. D.: 1971, Astrophys. J. 167, 499.

Dickens, R. J. and Saunders, J.: 1965, Roy. Observ. Bull., No. 101.

Geyer, E. H.: 1966, Mitt. Astron. Ges., No. 21, p. 122.

Geyer, E. H.: 1970, Astron. Astrophys. 4, 40.

Geyer, E. H.: 1971a, Mitt. Astron. Ges., No. 31, p. 168.

Geyer, E. H.: 1971b, IAU Colloquium No. 15, Veröff. Remeis-Sternwarte Bamberg 9, No. 100.

Geyer, E. H: 1973, in J. D. Fernie (ed.), Variable Stars in Globular Clusters and Related Systems, D. Reidel Publ. Co., Dordrecht, Holland, p. 88. 
Kukarkin, B. V.: 1973, in J. D. Fernie (ed.), Variable Stars in Globular Clusters and Related Systems, D. Reidel, Publ. Co., Dordrecht, Holland, p. 14.

Menzies, J. W.: 1967, thesis, unpublished.

Sawyer Hogg, H.: 1972, Comm. David Dunlap Obs., No. 346.

Sawyer Hogg, H.: 1973, Publ. David Dunlap Obs. 3, No. 6. 